\title{
Amplified imitation in percolation model of stock market
}

\author{
D. Makowiec ${ }^{\star}$, P. Gnaciński and W. Miklaszewski \\ Institute of Theoretical Physics and Astrophysics, Gdańsk University \\ ul.Wita Stwosza 57, 80-952 Gdańsk, Poland
}

The herd behavior of the Cont Bouchaud model is amplified by allowing clusters to copy decisions of some other cluster in the next time step. The results of the model are compared to data from Warsaw Stock Exchange. It follows that the mechanism of the amplified imitation could be responsible for the sell decision on a poorly developed, emergent market.

Keywords: Cont-Bouchaud stock market model, percolation, Monte Carlo simulations, fluctuation distribution, emergent stock markets

PACS code : 89.90. $+\mathrm{n} 05.40 .+\mathrm{j}$

\section{Introduction}

Over the last decade, artificial economic world living in computer made markets of financial assets has become an interest for physicists experienced in complex systems research. See [12 for review. The reasons for the ContBouchaud proposition is to explain effects of herd behavior which is observable among real traders. Differently from models of markets with agents rational, or heterogeneous, or adaptive, the Cont-Bouchaud model (CB-model, in short) considers noisy traders only, in the sense that traders do not have any rational strategy. Instead the strong communication and information links between traders is proposed. In effect agents form groups- called clusters, within which behavior of agants is common.

Complex system approach provides percolation as a powerful tool in study of connected objects of different type and origin, 34. The Cont-Bouchaud

\footnotetext{
ऋ fizdm@univ.gda.pl
} 
proposition is to adapt the percolation phenomena to design a stock market organization [5]. The original model uses infinite-range bond percolation. Similar interactions are proposed by considering nearest neighbor and site percolation instead 78,9] At a very few assumptions, the model reproduces, so-called stylized, facts known about fluctuations of price of real market assets which are widely accepted, [6]. In particular, the price fluctuations measured by so-called returns, i.e., change of the market price, (i) are not correlated, (ii) average of them is zero and (iii) tails of a distribution of returns decay accordingly to the power-law. These features are achieved only due to exploration of critical properties attributed to the percolation phenomena.

In the CB-model there is no other interaction between investors than instantaneous imitation through a cluster structure (these structure can change in time [10]). Our proposition is to consider imitation of one cluster by other clusters but taken in the next time step. We say the cluster imitates some other cluster with a time step delay. In this sense the herd behavior of the CB-model is amplified by the delay imitation.

The additional interaction introduced seems to be particularly suitable when poorly developed stock markets are considered. In the result of multi century experience the large markets, like London City or New York Stock Exchange, have developed the solid structures and their regulations are firmly executed. One can say that the fundamental assumption of Efficient Market Hypothesis 11] that the whole information is available for all investors at the same instant, is close to be satisfied. The race and competition between individual investors effects in that the same information is differently understood and in consequence differently transferred into market decisions. There is no place for imitation between investors for at least two reasons. First, the next piece of information arrives at the market and investors are forced to concentrate on this new subject. Second, investors are known to be extremely strong individuals in the sense it is said, they are overconfident about their personal understanding of the market. Such attitude leads to the strong disagreement between investors rather than cooperation [12. However when a market is far from equilibrium, because, for example, a market is strongly influenced by political decisions or liquidity of a stock is limited then the only strategy to win is to search for 'well informed' investors to join their team or if it is impossible to follow their decisions. 
In the subsequent sections we provide: setting of the model (Section 2), analysis of results obtained in simulations of the model (Section 3), examples of stocks data from the Warsaw Stock Exchange market for which the hypothesis of amplified imitation as underlying mechanism of driving the price can be proposed (Section 4). In the closing section further possible modifications to the model are proposed.

\section{Details of the model}

In the Stauffer et al. representation [789] of the CB-model one deals with a number of investors who are distributed on a square lattice of a linear size $L$. Each of the investors is assigned to a site at random with the probability $p$. A site can be occupied by one investor or left empty. The geometric relations arising from nearest neighboring occupied sites, called clusters, are interpreted as coalitions of investors- groups of traders that act together on a stock market. The coalitions last for a limited period of time after which a new arrangement of stock market participants is introduced. The clusters take decisions randomly and independently of each other to buy or sell, with the probability $a$, or just wait with a probability $1-2 a$. The resulting stock price is proportional to the sum of demand and sell orders from all clusters which have been active during a time step. The parameter $a$ is called activity and can be seen as a control of the length of a time step [8]. If activity is high, which means that $a$ is close to 0.5 , then all clusters are active during a session. Such activity characterizes a long time step. The independent and stochastic decisions taken during long sessions lead to the histogram of price changes of a Gaussian type. If the activity is low, $a<\frac{1}{L^{2}}$, what means that a time step is short, then on average one cluster acts during a given market session. In effect the histogram of price changes reflects properties of the cluster size distribution. If $p$ is close to the percolation threshold then the power-law decay of tails of the distribution is observed with the exponent $\tau=2$ in case of a square lattice [3].

Let investors occupy randomly a fraction $p_{c}=0.592746$ of the sites of $L \times$ $L$ square lattice. This particular value of the parameter $p_{c}$ provides critical properties for a cluster structure on a square lattice: a cluster that spans the 

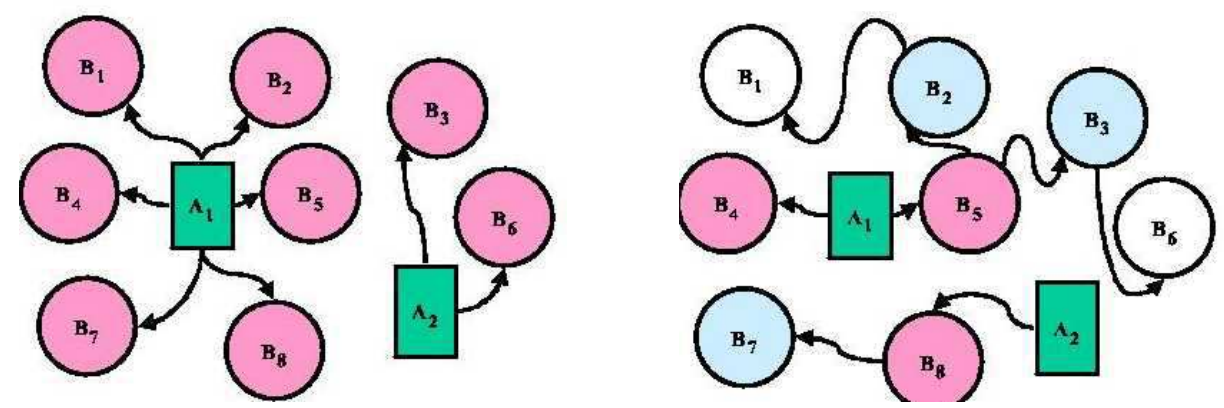

Fig. 1. The network of imitation between clusters- an illustration. Green (dark in black and white view) rectangles represent clusters of the class A and circles represent clusters of the class $\mathrm{AB}$ - less red color (less gray) means longer delay time in imitation; left panel: trees of the model A; right panel: trees of the model $\mathrm{AB}$.

lattice occurs with the probability $\frac{1}{2}$. In the following we use the HoshenKopelman algorithm [13, to identify clusters.

The clusters of size greater than 2 are split into two classes:

- class A contains clusters of well informed agents. These clusters take decisions independently and at random: they buy or sell at the probability $a$ and at probability $1-2 a$ they do nothing.

- class B contains clusters which imitate other clusters. Each cluster is linked to some other cluster. Imitation means that the next time step decision of a B-class cluster is exactly the same as the present decision of the cluster to which the B-class cluster is linked.

Let us assume that all clusters of size smaller than 3 run the ordinary CBmodel dynamics.

The following two types of imitation will be considered: model A - each cluster from the class $\mathrm{B}$ at random chooses a cluster from the class $\mathrm{A}$ to imitate, and model AB - each cluster from the class B is linked to a randomly choosen cluster from any class. So that the pool of clusters is arranged into trees, see Fig. 11. The root of each tree is a leader- an independently acting participant of a market. The rest of clusters grupped in a tree is either of one step depth tree - case of the model A, or many steps depth tree - case of the model AB. Clusters from a tree take the same market decision as their leader but with a delay which equals to the path length to the leader of the tree.

Let us introduce the parameter $b \in(0,1]$ the probability that a cluster of size greater than 2 belongs to the class A. At a given $b$ the average number of 
clusters in one tree is

$$
1+\frac{(1-b) N}{b N}=\frac{1}{b}
$$

where $N$ is the average number of clusters of size greater than 2. Hence if $b=1$ then both models collapse to the original CB-model.

\section{Results}

Our simulations are performed on a square lattice of the linear size $L=200$. On this lattice at $p_{c}$ the average number of clusters of size greater than 2 is $391 \pm 25$ (total number of all clusters of 1 's is $1205 \pm 50$ ). Setting $b=0.001$ indicates that at most one tree is present on the lattice while $b=0.005(b=0.01)$ allows two (four, respectively) trees to exist on the lattice. If $b=0.1$ then at average about 40 trees take part in the market game. Therefore, very long runs must be considered to obtain satisfactory statistics. Our simulations are performed for (number of lattice rearrangements) * (length of each of arrangement $)=10^{6} * 200$ time steps and experiments were repeated several times.

Let us start with presenting examples of time series emerging from the two microdynamics studied, Fig. 2. The activity $a=0.001$ in all experiments. This value of activity has been found as satisfactory low to be sensitive to observe critical cluster distribution, i.e., the power-law decay of distribution tails. [89]. At average the activity of the whole market is constants, but existing tree structures lead to strongly synchronized actions. In effect we observe huge impacts on a price change after each decision done by a member of the class $\mathrm{A}$ if there are only few trees on a lattice, see Fig. 2(a) and Fig. 2(b). In case of the model $\mathrm{A}$ isolated high changes of a price are presents. In case of the model AB an avalanche of price changes developes. If the number of leaders grows, see Fig. 2(c), trees of dependencies are smaller and values of returns go

down. However we still observe bursts of activity. The volatility, by means the 

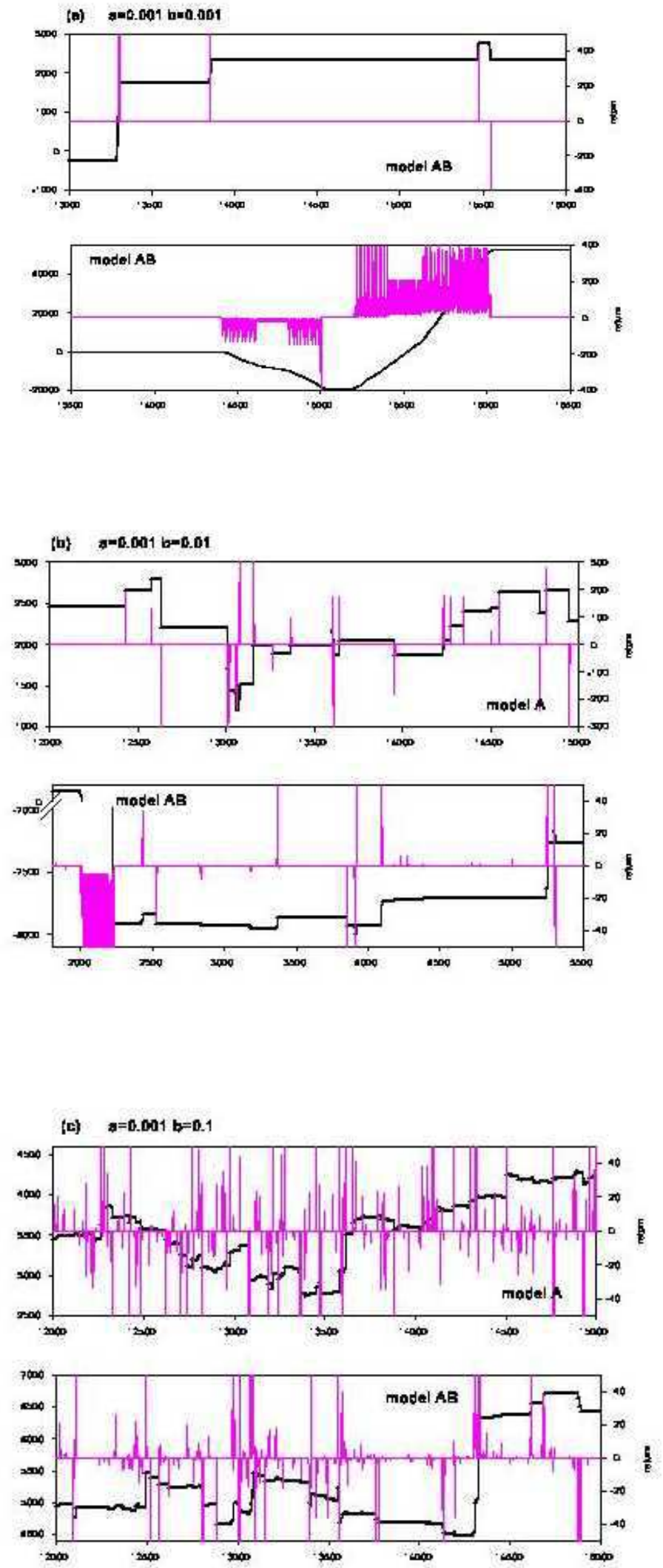

Fig. 2. Examples of series of price and returns arising from both models if (a) $b=0.001$, (b) $b=0.01$, (c) $b=0.1$. 


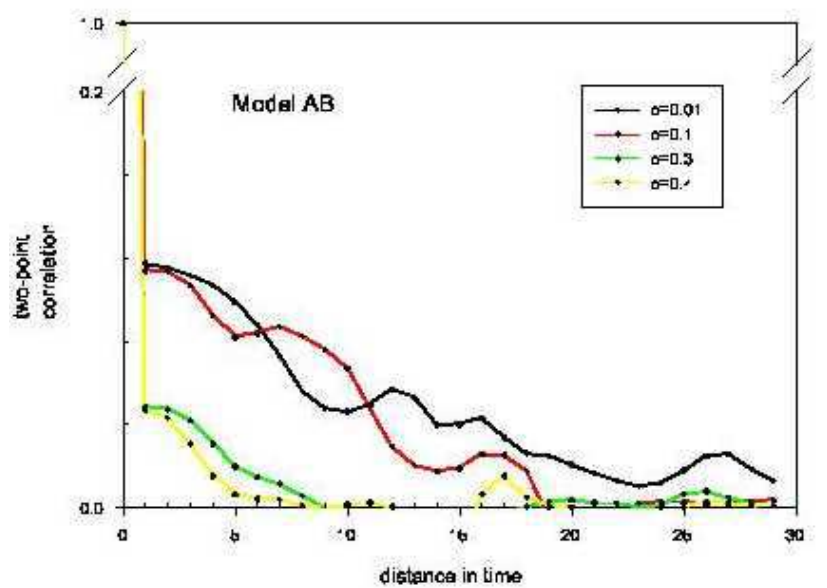

Fig. 3. Two-point autocorrelation function for the model AB for different values of $\mathrm{b}$

standard deviation, of the recorded data for different values of $b$ is as following:

$\begin{array}{lrr}b & \text { model } A & \text { model } A B \\ 0.001 & 21.2 & 31.2 \\ 0.005 & 38.5 & 32.6 \\ 0.01 & 39.5 & 30.7 \\ 0.1 & 21.8 & 20.4 \\ 0.5 & 19.5 & 19.4 \\ 1.0 & 19.3 & 19.3\end{array}$

So that one can say that for $b>0.1$ the volatility in both systems is close to the volatility of the pure CB-model.

It occurs that the two-point autocorrelation function decays sharply in 1 step independently of $b$ as far as the model $\mathrm{A}$ is considered. However in case of the model $\mathrm{AB}$ the presence of delay trees transmits into weak correlation. We observe nonzero correlation but $<0.2$ for few time steps if $b<0.4$, see Fig. [3.

On Fig. 4 we show distributions of normalized returns. The normalization means dividing each return by the standard deviation. The synchronized decisions of clusters result in huge returns and what follows in fat wings of the return distribution. However the distribution arisen from the model A points at the absence of small returns also. Close to the zero-return peak, symmet- 

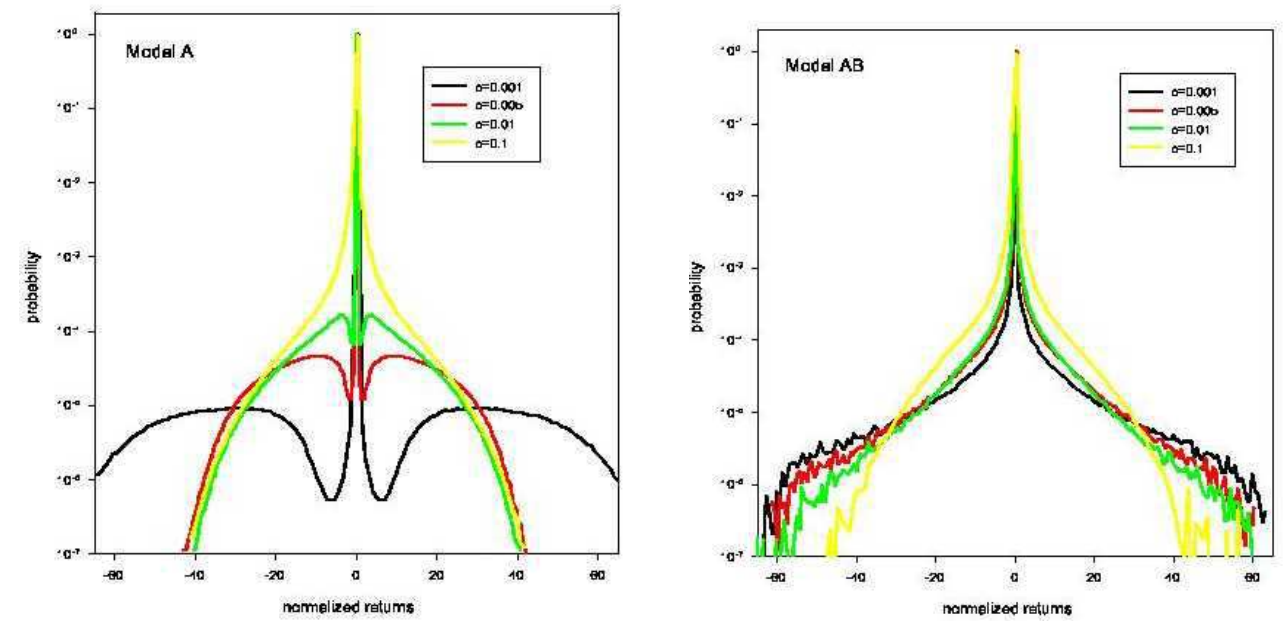

Fig. 4. Distribution of normalized returns for both models considered. Left panel: model A. Right panel: model AB. Notice really huge returns observed if $b$ is small enough and two symmetric dips around the zero-return peak in case of the curve associated to the model A. Tails of the distributions with $b>0.1$ decay accordingly to the power-law with exponent about 1.8

rically on its both sides, we observe two minimums. But with $b \geq 0.1$ both models lead to distributions of returns hardly distinguishable from the CB model distribution. It seems that effect on a price caused by actions of more than 40 independent groups of investors is similar to that ones which are undertaken by the whole 400 independent investors possible to exists on $200 \times 200$ lattice.

\section{$4 \quad$ Polish stock market and imitation}

We have examined 73 series of prices of stocks from Polish stock market to find signs of the model A: dips around the zero-return peak, or the model AB: fat tails. We analysed the date which were collected during last six or seven years. The Warsaw Stock Exchange regulations on the minimal price change imply that the size of a histogram bin has to be carefully selected. In the following figures we plot distributions of logarithmic returns, namely, return(t)= log price(t) -log price (t-1), which are normalized by their standard deviation. The minimal price change rule leads to the separation from the return zero less than 0.064. Therefore we decided that the value 0.20 for the size for a histogram bin is proper. Accordingly to the distribution shape the stocks have 

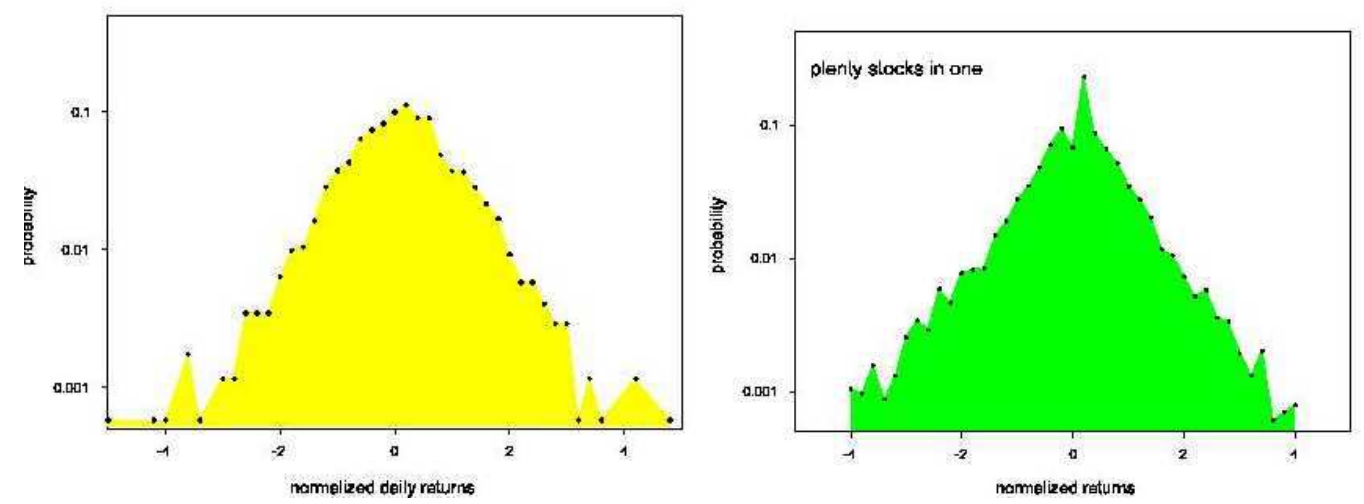

Fig. 5. Left panel: distribution of returns of Warsaw stock index, right panel: distribution of returns of stocks classified as irregular

been divided into two groups: regular - with the distribution similar to the distribution of WIG- the Warsaw Stock Exchange market index, and others, see Fig. 5. Let us point at the minimum on the left side of the zero-return peak. To identify stocks with irregular distribution we searched for the zero-returns properties and fat tails. Series classified as possible examples which exhibit properties of the model A and the model AB are collected in Fig. 6. Together we show time series of these stocks. These stocks represent weak firms: low capital, low liquidity, often at the edge of the bankruptcy. The DFA method 14] for quantification of long-termed correlation in time-series provides the crossover to the antipersistency behavior for long time distances. 40 stocks of all 73 considered by us are found with the corresponding exponent $\alpha<0.48$. Among them 19 shows $\alpha<0.40$.

\section{Closing}

Presented results are only preliminary and further investigations should be undertaken. The proposed microdynamics leads to the non-realistic global effect in a sense that each price change is accepted. In real markets there are rules preventing from unlimited large or infinitely small changes. Nevertheless, the observed on the Polish market time series justify the modifications introduced by us to the original CB-model. Specially when emerging markets are considered then the assumption about imitating others in place of undertaking own reasoning seems to be legalized. However one must be careful in a quantitative conclusion suggested when comparing the model A distribution to a real series 

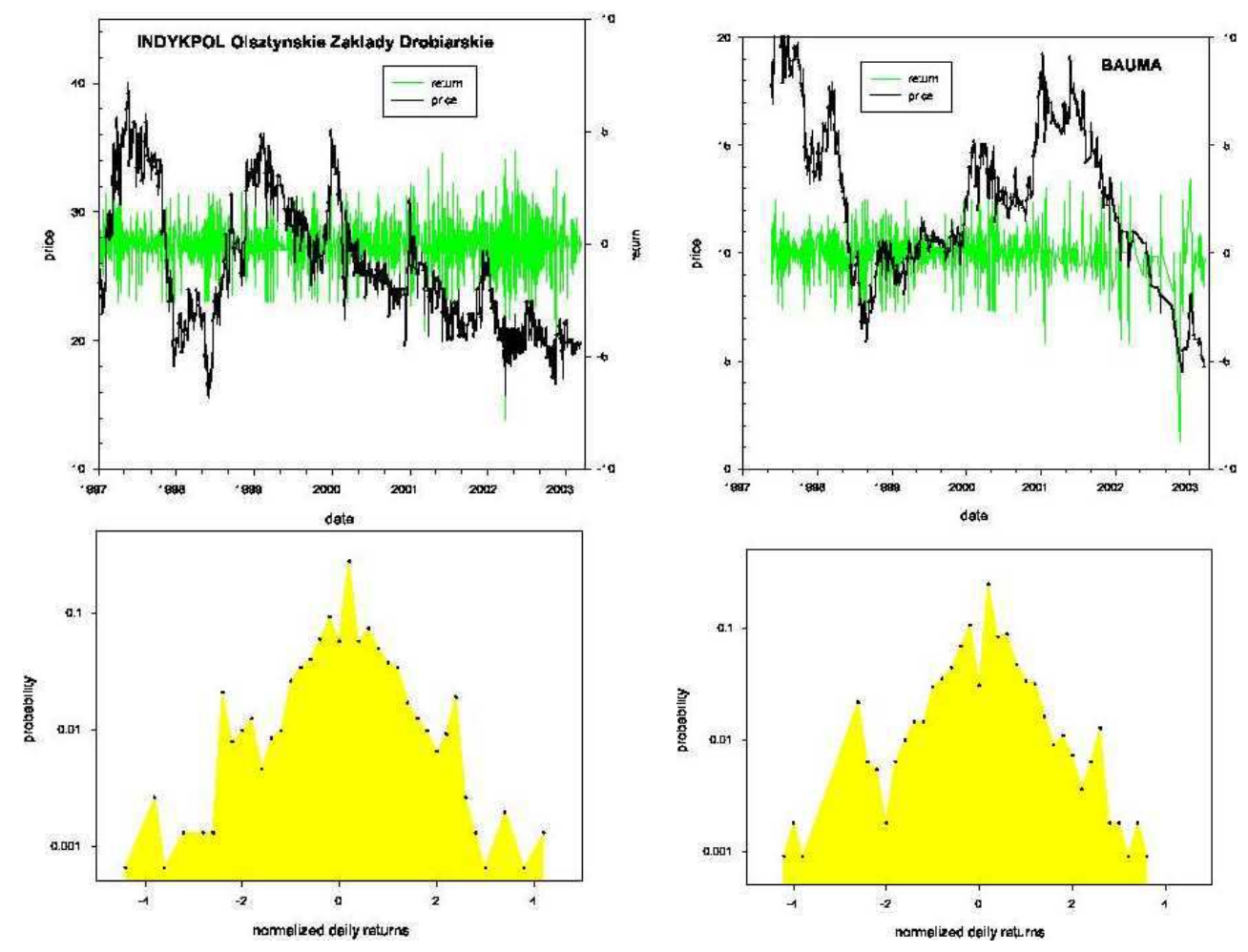

Fig. 6. Time series of stocks which lead to distribution of returns irregular. Both firms are small. DFA gives: 0.329 for Indykpol and 0.408 for Bauma

distribution that a stock is driven by not more than a few trees of investors.

There are some natural possibilities for further model development. The existing extension of the $\mathrm{CB}$ model such as adjusting activity with respect to the latest price movements [15] offers the straightforward way. The next possibility is to inject imitating agents, agents of the class $B$, into different from the CB model dynamics. For example, it should be interesting to consider the Lux-Marchesi model [16] with speculative investors divided into imitation trees. Finally, one should consider switching imitating agents into overconfident investors, which are know to be present on well developed markets [12]. Such investors being overconfident about own reasoning, understand better a market than other investors. Therefore each of overconfident investors can predict with certainty what some other investor will do at the next time step. Having such a knowledge he modifies his present decision. Thus, he buys now if the other investor will buy, he sells if the other investor will sell. Simulations with described microdynamics are performed and soon will be presented.

\section{Acknowledgement}


We are grateful to Professor Ditrich Stauffer for the source code of ContBouchaud model. This work is supported by Gdańsk University: BW 5400-50014-3. The simulations, partially, have been performed in TASK- Academic Computer Center in Gdansk.

\section{References}

[1] M. Levy, H. Levy and S. Solomon, Microscopic Simulation of Financial Markets, Academic Press, New York, 2000.

[2] E. Samanidu, E. Zschischang, D. Stauffer and T. Lux : Microscopic Models of Financial Markets, in F. Schweitzer (ed.), Microscopic Models for Economic Dynamics Lecture Notes in Physics, Springer, Berlin-Heidelberg 2002.

[3] D. Stauffer and A. Aharony, Introduction to Percolation Theory, 2nd Edition, Taylor\&Francis, London, 1992.

[4] H. E. Stanley, J. S. Andrade Jr, S. Havlin, H. A. Makse and B. Suki, Physica A 266 (1999) 5-16: Percolation phenomena: a broad-brush introduction with some recent applications to porous media, liquid water and city growth.

[5] R. Cont and J. P. Bouchaud, Macroeconomic Dynamics 4 (200) 170: Herd behaviour and aggregate fluctuation in financial markets.

[6] R. Cont, Quant. Finance 1 (2001) 223-226: Empirical properties of asset returns: stylized facts and statistics issues.

[7] D. Stauffer and D. Sornette, Physica A 252 (1998) 271-277: Log-periodic oscillations for biased diffusion on random lattice

[8] D. Stauffer and T. J. P. Penna, Physica A 256 (1998) 284-290: Crossover in the Cont-Bouchaud percolation model for market fluctuation.

[9] F. Castiglione and D. Stauffer, Physica A 300 (2001) 531-538: Multi-scaling in the Cont Bouchaud microscopic market model.

[10] V. M. Eguiluz and M. G. Zimmermann, Phys.Rev.Lett. 85 (2000) 5659-5662: Transmission of Information and herd behavior: An Application to Financial Markets.

[11] E. F. Fama, J.Finance 25 91970) 383-417: Efficient capital markets. A review of theory and empirical work.

[12] M. Harrison and D. Kreps, Quarterly Journal of Economics 92 (1978) 323336: Speculative investor behavior in a stock market with heterogeneous expectations,

J. Scheinkman and Wei Xiong, Overconfidence and Speculative Bubles, WEHIA Triest preprint (2002) 
[13] J. Hoshen and R. Kopelman, Phys. Rev. B 14 (1976) 3438-3445: Percolation and cluster distribution. I. Cluster multiple labelling technique and critical concentration algorithm.

[14] C. K. Peng, S. Havlin, H. E. Stanley and A. L. Goldberg, Chaos 5 (1995) 82-87: Quantification of scaling exponents and crossover phenomena in nonstationary heartbeat time series.

[15] D. Stauffer, P. M. C. de Oliveira and A. Bernardes, Int. J. Theor. and Appl. Finance 2 (1999) 83-94: Monte Carlo simulation of volatility clustering in market model with herding.

[16] T. Lux and M. Marchesi, Nature 397 (1999) 498-500: Scaling and criticality in a stochastic multiagent model of a financial market. 